\title{
Specimen records for North American Lepidoptera (Insecta) in the Oregon State Arthropod Collection. Hepialidae Stephens, 1829
}

Jon H. Shepard

Paul C. Hammond

Christopher J. Marshall

Oregon State Arthropod Collection, Department of Integrative Biology, Oregon State University, Corvallis OR 97331

Cite this work, including the attached dataset, as:

Shepard, J. H., P. C. Hammond, C. J. Marshall. 2019. Specimen records for North American Lepidoptera (Insecta) in the Oregon State Arthropod Collection. Hepialidae Stephens, 1829. Catalog: Oregon State Arthropod Collection 3(2) (beta version). http:// dx.doi.org/10.5399/osu/cat osac.3.2.4590

\section{Introduction}

These records were generated using funds from the LepNet project (Seltmann et. al., 2017) - a national collaborative effort to create digital records for North American Lepidoptera. The dataset published herein contains the label data for all North American specimens of Hepialidae residing at the Oregon State Arthropod Collection as of March 2019. A beta version of these data records will be made available on the OSAC server (http: / / osac.oregonstate.edu/IPT) at the time of this publication. The beta version, entitled, OSAC_Heplialidae_2019_ver_beta will be replaced in the near future with an official release (OSAC_Hepialidae_2019_ver_1.0), which will then also be archived as a supplemental file to this publication.

\section{Methods}

Basic digitization protocols and metadata standards can be found in (Shepard et al. 2018). Identifications were made by Jon Shepard with reference to an unpublished key to the PNW Hepialidae Wagner (1983). Identification and nomenclature were checked against the Digital Guide to Moth Identification (Moths Protographers Group, 2019).

\section{Results}

As of March 2019, the collection possessed 70 specimens of North American Hepialidae, belonging to the genera: Sthenopis Pagenstecher, 1909, Phymatopus Wallengren, 1869 and Gazoryctra Hübner, 1820 (Table 1)

Additional material from outside of North America is also present in the holdings, but was not digitized and catalogued at this time.

Label data for these specimens can be accessed via online data portals that digest OSAC IPT data directly (e.g, www.gbif.org) or by accessing the archived datasets stored in the supplemental files of this paper. 
Table 1. Species inventory of OSAC Hepialidae

Taxon \# specimens

\begin{tabular}{|c|c|}
\hline Sthenopis purpurascens Packard, 1863 & 3 \\
\hline Sthenopis argenteomaculatus Harris, 1841 & 1 \\
\hline Phymatopus hectoides (Boisduval, 1868) & 12 \\
\hline Phymatopus californicus (Boisduval, 1868) & 7 \\
\hline Phymatopus behrensi (Stretch, 1872) & 3 \\
\hline Phymatopus (undetermined) & 2 \\
\hline Gazoryctra novigannus (Barnes and Benjamin, [1926]) & 16 \\
\hline Gazoryctra mathewi (W.H. Edwards, 1874) & 18 \\
\hline Gazoryctra (undetermined) & 8 \\
\hline total & 70 \\
\hline
\end{tabular}

\section{Discussion}

Minor corrections and other small modifications made in the future to this dataset will be accomplished by issuing a new version and providing a file of modified records to the supplemental files. Major additions, such as new records, new data fields, new contributors or other significant modifications will be accomplished through the generation and release of new datasets.

\section{Acknowledgments}

The records published herein were generated with funds from NSF: DBI\# 1601888: Digitization TCN: Collaborative Research: Lepidoptera of North America Network: Documenting Diversity in the Largest Clade of Herbivores.

\section{References}

Moth Photographers Group (2019) Digital Guide to Moth Identification.

https: / / mothphotographersgroup.msstate.edu [accessed April 1, 2019].

Seltmann, K.C. et al. 2017. LepNet: The Lepidoptera of North America Network. Zootaxa 4247 (1):

073-077. https:/ / doi.org/10.11646/ zootaxa.4247.1.10.

Shepard J.H., C.J. Marshall, P.C. Hammond. 2018. North American Lepidoptera in the Oregon State Arthropod Collection: Hesperiidae, Pieridae, Papilionidae. Catalog: Oregon State Arthropod Collection 2(2): 1-2 and supplement file. doi: http:// dx.doi.org/10.5399/osu/cat osac.2.2.4480

Wagner, D. 1983. Hepialidae of the Pacific Northwest pp. [1]-18. unpublished manuscript. 